\title{
Computers
} \& Structures

\section{Calculation of derivative of complex modes using classical normal modes}

\author{
Sondipon Adhikari* \\ Department of Engineering, University of Cambridge, Trumpington Street, Cambridge CB2 1PZ, UK \\ Received 19 March 1999; accepted 8 December 1999
}

\begin{abstract}
In this paper, a method to calculate derivatives of eigenvectors of damped discrete linear dynamic system with respect to the system parameter is presented. Due to the non-proportional nature of the damping, the eigenvectors become complex, and as a consequence, the derivatives also become complex. The derivatives are calculated using small damping assumption, and the method avoids using the state-space approach. The results are obtained in terms of complex modes and frequencies of the second-order system, which in turn are related to the eigensolutions of the undamped system using perturbation method. Based on the derivatives, an expression for total change of the complex eigenvectors is obtained for a more general case when all the elements of mass, stiffness and damping matrices are varying. Application and accuracy of the derived expressions are demonstrated by considering numerical examples. (c) 2000 Elsevier Science Ltd. All rights reserved.
\end{abstract}

Keywords: Sensitivity; Complex modes; Non-proportional damping; Derivative of eigensolutions; Perturbation methods

\section{Introduction}

In structural design/optimization and system identification studies, derivatives of eigenvectors provide important information. Eigenvector derivatives also constitute a central role in the analysis of stochastic dynamic systems. Several papers exist on derivatives of eigensolutions of undamped system. In one of the earliest works, Fox and Kapoor [1] have given exact expressions for derivatives of eigenvalues and eigenvectors with respect to any design variable. The eigenvector derivatives were obtained in terms of changes in the system property matrices and all eigenvectors of the structure in its current state, and have been used extensively in a wide range of application areas of structural dynamics. Later, many authors have extended this kind of sensitivity analysis to include asymmetry of the system and to the systems with repeated eigenvalues.

\footnotetext{
${ }^{*}$ Fax: +44-1223-332662.

E-mail address: sa225@eng.cam.ac.uk (S. Adhikari).
}

Plaut and Huseyin [2] have extended Fox and Kapoors method to the system with general mass and stiffness matrices. Nelson [3] proposed an efficient method to calculate eigenvector derivative which requires only the eigenvalue and eigenvector under consideration. Adelman and Haftka [4] have given a comprehensive review of research on sensitivity analysis of discrete structural systems. Murthy and Haftka [5] have surveyed the methods for sensitivity analysis of the eigenvalue problem associated with general (non-Hermitian) matrix.

The above-mentioned analytical methods are based on the undamped free vibration of the system. For damped systems, it is well known that unless the damping matrix of the structure is proportional to the inertia and/or stiffness matrices (proportional damping) or can be represented in the series form derived by Caughey [6], the mode shapes of the system will not coincide with the undamped mode shapes. In the presence of general nonproportional viscous damping, the equations of motion in the modal coordinates will be coupled through the offdiagonal terms of the modal damping matrix, and the mode shapes of the system will in general be complex. 
Apart from the mathematical reason, while conducting experimental modal analysis also one often identifies complex modes (see e.g. Ref. [7]). So, for many application areas of structural dynamics which utilize the eigensolution derivatives, e.g. modal updating, damage detection, design optimization and stochastic finite element methods, sensitivity of complex eigensolutions should be incorporated in the analysis in order to take damping into consideration. Most of the available methods to obtain the derivatives of the complex eigensolutions employ first-order formulation of the equations of motion (see, e.g. Refs. [8-10]). However, first-order or 'Hamiltonian' methods have never been popular to solve engineering dynamic problems. For this reason, the expressions for derivative of the complex modes available in the literature have not been utilized in practice.

In this paper, we determine derivative of complex modes with respect to some set of design variables in non-proportionally damped discrete linear systems. The approach taken here avoids the use of state-space formulation and is consistent with the notion of traditional modal analysis methods. Before going into the detailed derivation of derivative of the complex eigenvectors, the classical first-order perturbation-based analysis to determine complex eigensolutions under the small damping assumption is briefly described in Section 2. It is indicated that a minor modification of the known expression can give more accuracy. In Section 3, derivatives of eigenvectors are derived approximately using a small damping assumption. With these rates, total changes in eigenvectors are obtained in Section 4 for a more general case when all the elements of mass, stiffness and damping matrices are perturbed. In Section 5, a two degree-of-freedom system which shows the 'curve-veering' phenomenon has been considered to illustrate the application of the expression for rates of changes of eigenvectors. Numerical accuracy of the approximate expression for derivative of eigenvectors is also examined by considering a 10 degree-of-freedom linear springmass system.

\section{Complex eigenvalues and eigenvectors}

The equations of motion for free vibration of a linear damped discrete system with $N$ degrees of freedom can be written as

$\mathbf{M} \ddot{\mathbf{y}}+\mathbf{C} \dot{\mathbf{y}}+\mathbf{K y}=0$,

where $\mathbf{M}, \mathbf{C}$ and $\mathbf{K}$ are $N \times N$ mass, damping and stiffness matrices, respectively, and $\mathbf{y}$ is the $N \times 1$ vector of the generalized coordinates. We seek a harmonic solu- tion of the form $\mathbf{y}=\mathbf{u} \exp [i \lambda t]$, where $\mathrm{i}=\sqrt{-1}$ is the unit complex number. Substitution of $\mathbf{y}$ in Eq. (1) yields

$-\lambda^{2} \mathbf{M u}+\mathrm{i} \lambda \mathbf{C u}+\mathbf{K u}=0$.

This equation is satisfied by the $i$ th latent root (natural frequency), $\lambda_{i}$, and $i$ th latent vector (mode shape), $\mathbf{u}_{i}$, of the $\lambda$-matrix problem (see Ref. [11]), so that

$-\lambda_{i}^{2} \mathbf{M u}_{i}+\mathrm{i} \lambda_{i} \mathbf{C u}_{i}+\mathbf{K} \mathbf{u}_{i}=0 \quad \forall i=1, \ldots, N$.

Unless $\mathbf{C}$ is simultaneously diagonalizable with $\mathbf{M}$ and $\mathbf{K}$ (conditions for which was derived by Caughey and O'Kelly [12]), in general $\lambda_{i}$ and $\mathbf{u}_{i}$ will be complex. The procedures to obtain the complex eigensolutions follow mainly two routes: the state-space method and approximate methods in ' $N$-space'. The state-space method (see Ref. [13]) although exact in nature requires significant numerical effort for obtaining the eigensolutions as the size of the problem doubles. Moreover, this method also lacks some of the intuitive simplicity of traditional modal analysis. For these reasons, there has been considerable research effort to calculate the complex eigensolutions of non-proportionally damped structures in $\mathrm{N}$-space. Using first-order perturbation analysis, Rayleigh [14] considered approximate methods to determine $\lambda_{i}$ and $\mathbf{u}_{i}$ by assuming that the elements of $\mathbf{C}$ are small but otherwise general. Cronin [15] has given a power series expression of eigenvalues and eigenvectors by using perturbation method. Recently, Woodhouse [16] has extended Rayleigh's analysis to the case of more general linear damping models described by convolution integrals of the generalized coordinates over the damping kernel functions. Following a similar line of analysis, Bhaskar [17] developed a procedure to exactly obtain $\lambda_{i}$ and $\mathbf{u}_{i}$ by an iterative method and has shown that the results from the classical perturbation analysis can be obtained as a special case. For later reference, we briefly outline the procedure to obtain them.

Consider the undamped natural frequencies $\omega_{i}$ and mode shapes $\mathbf{x}_{i}$ satisfying

$\mathbf{K} \mathbf{x}_{i}=\omega_{i}^{2} \mathbf{M} \mathbf{x}_{i} \quad \forall i=1, \ldots, N$.

The mode shape vectors are normalized in a usual way so that

$\mathbf{x}_{j}^{\mathrm{T}} \mathbf{M} \mathbf{x}_{i}=\delta_{j i}, \quad \mathbf{x}_{j}^{\mathrm{T}} \mathbf{K} \mathbf{x}_{i}=\omega_{i}^{2} \delta_{j i} \quad \forall j, i=1, \ldots, N$,

where $\delta_{j i}$ is the Kroneker's delta function and $(\bullet)^{\mathrm{T}}$ denotes matrix transpose. Since $\mathbf{x}_{i}, \forall i=1, \ldots, N$ form a complete set of vectors, we can expand $\mathbf{u}_{i}$ as a complex combination of $\mathbf{x}_{i}$. In the case when the entries of the $\mathbf{C}$ matrix are not very big, the roots of Eq. (3), $\lambda_{i}$, will be close to those of Eq. (4), $\omega_{i}$, and the corresponding eigenvectors, $\mathbf{u}_{i}$, are also expected to be close to $\mathbf{x}_{i}$. Thus, we can try a solution of the form 
$\mathbf{u}_{i}=\sum_{j=1}^{N} \alpha_{j}^{(i)} \mathbf{x}_{j} \quad$ where $\alpha_{i}^{(i)}=1$ and $\left|\alpha_{j}^{(i)}\right| \ll 1 \forall j \neq i$

Substituting $\mathbf{u}_{i}$ in Eq. (3), premultiplying by $\mathbf{x}_{k}^{\mathrm{T}}$ and using the orthogonality properties of the undamped mode shapes described by Eq. (5), one obtains

$-\lambda_{i}^{2} \alpha_{k}^{(i)}+\mathrm{i} \lambda_{i} \sum_{j=1}^{N} \alpha_{j}^{(i)} C_{k j}^{\prime}+\omega_{k}^{2} \alpha_{k}^{(i)}=0$,

where $C_{k j}^{\prime}=\mathbf{x}_{k}^{\mathrm{T}} \mathbf{C} \mathbf{x}_{j}$ are the elements of the damping matrix in modal coordinates. The $i$ th equation of this set can be obtained by setting $k=i$ : now neglecting the second-order terms involving $\alpha_{j}^{(i)}$ and $C_{i j}^{\prime}, \forall j \neq i$, and also noting that $\alpha_{i}^{(i)}=1$, Eq. (7) yields

$-\lambda_{i}^{2}+\mathrm{i} \lambda_{i} C_{i i}^{\prime}+\omega_{i}^{2}=0, \quad$ or $\quad \lambda_{i} \approx \omega_{i}+\mathrm{i} C_{i i}^{\prime} / 2$,

which is the approximate expression for the complex natural frequencies. Again Eq. (7) can be rewritten as

$$
\begin{aligned}
& -\lambda_{i}^{2} \alpha_{k}^{(i)}+\mathrm{i} \lambda_{i}\left(\alpha_{i}^{(i)} C_{k i}^{\prime}+\alpha_{k}^{(i)} C_{k k}^{\prime}+\sum_{j \neq k \neq i}^{N} \alpha_{j}^{(i)} C_{k j}^{\prime}\right) \\
& +\omega_{k}^{2} \alpha_{k}^{(i)}=0,
\end{aligned}
$$

which leads to

$\alpha_{k}^{(i)} \approx-\frac{\mathrm{i} \lambda_{i} C_{k i}^{\prime}}{\omega_{k}^{2}-\lambda_{i}^{2}+\mathrm{i} \lambda_{i} C_{k k}^{\prime}}$

By replacing $\omega_{k}$ from Eq. (8) for the $k$ th set, the denominator appearing in the above expression can be factorized as

$\omega_{k}^{2}-\lambda_{i}^{2}+\mathrm{i} \lambda_{i} C_{k k}^{\prime} \approx-\left(\lambda_{i}-\lambda_{k}\right)\left(\lambda_{i}-\lambda_{k}^{*}\right)$,

where $(\bullet)^{*}$ denotes complex conjugation. With the help of this factorization, Eq. (10) can be expressed as

$\alpha_{k}^{(i)} \approx \frac{\mathrm{i} \lambda_{i} C_{k i}^{\prime}}{\left(\lambda_{i}-\lambda_{k}\right)\left(\lambda_{i}-\lambda_{k}^{*}\right)}$

and consequently, from the series sum of Eq. (6), the approximate expression for the complex modes reads

$\mathbf{u}_{i} \approx \mathbf{x}_{i}+\sum_{k=1}^{N} \frac{i \lambda_{i} C_{k i}^{\prime} \mathbf{x}_{k}}{\left(\lambda_{i}-\lambda_{k}\right)\left(\lambda_{i}-\lambda_{k}^{*}\right)}$.

The approach taken here is very similar to the classical perturbation analysis $[14,16]$, but the $\alpha_{k}^{(i)}$ expressed by Eq. (12) appear to be slightly different. The classical expression for this is, $\alpha_{k}^{(i)} \approx \mathrm{i} \omega_{i} C_{k i}^{\prime} /\left(\omega_{i}^{2}-\omega_{k}^{2}\right)$, which is equivalent to replacing the complex natural frequencies by undamped natural frequencies in Eq. (12). Numerical calculations using the $\alpha_{k}^{(i)}$ described by Eq. (12) yield more accurate results than those of the classical analysis. To illustrate this, consider the simple example of the two degree-of-freedom system shown in Fig. 1. For the parameter values selected here (see figure caption), the damping is quite high: when $k_{2}=1.1 k_{1}$, the $Q$-factors for the two modes are $Q_{1} \approx 152.601$ and $Q_{2} \approx 9.3754$. Using Eq. (13), the approximate complex mode shapes can be calculated as

$$
\begin{aligned}
& \mathbf{u}_{1}=\left[\begin{array}{c}
0.7870-0.0661 \mathrm{i} \\
0.6287+0.1070 \mathrm{i}
\end{array}\right], \\
& \mathbf{u}_{2}=\left[\begin{array}{c}
-0.6406-0.1179 \mathrm{i} \\
0.7797-0.0729 \mathrm{i}
\end{array}\right] .
\end{aligned}
$$

The corresponding exact mode shapes from the statespace formulation can be obtained as

$$
\begin{aligned}
& \mathbf{u}_{1}^{\mathrm{e}}=\left[\begin{array}{c}
0.7870-0.0661 \mathrm{i} \\
0.6443+0.1018 \mathrm{i}
\end{array}\right], \\
& \mathbf{u}_{2}^{\mathrm{e}}=\left[\begin{array}{c}
-0.6406-0.1179 \mathrm{i} \\
0.7631-0.0603 \mathrm{i}
\end{array}\right],
\end{aligned}
$$

while the classical perturbation method gives

$$
\begin{aligned}
& \mathbf{u}_{1}^{\mathrm{c}}=\left[\begin{array}{c}
0.7870-0.0661 \mathrm{i} \\
0.4658+0.2362 \mathrm{i}
\end{array}\right], \\
& \mathbf{u}_{2}^{\mathbf{c}}=\left[\begin{array}{c}
-0.6406-0.1179 \mathrm{i} \\
0.8866-0.3989 \mathrm{i}
\end{array}\right] .
\end{aligned}
$$

For comparison, the modes are normalized to have the same numerical value in the first element, so that only the second element differs. It is clear that the results obtained from the expression suggested here are closer to the exact values than the classical analysis. Beside this, the expression in Eq. (13) also indicates a conceptual difference from the classical analysis: unlike there, the 'correction terms' no longer remain purely imaginary. This in turn means that the real part of the complex mode shapes are not the undamped mode shapes. This fact is indeed true and can be verified from the exact analysis.

\section{Rates of change of eigenvectors}

Suppose the structural system defined in Eq. (1) can be described by a set of $m$ parameters (design variables), $\mathbf{g}=\left\{g_{1}, g_{2}, \ldots, g_{m}\right\}^{\mathrm{T}}$, so that the mass, damping and stiffness matrices become functions of $\mathbf{g}$, that is, $\mathbf{M} \equiv \mathbf{M}(\mathbf{g}), \mathbf{C} \equiv \mathbf{C}(\mathbf{g})$ and $\mathbf{K} \equiv \mathbf{K}(\mathbf{g})$. Assume further that the design variables undergo a small change of the form $\Delta \mathbf{g}=\left\{\Delta g_{1}, \Delta g_{2}, \ldots, \Delta g_{m}\right\}^{\mathrm{T}}$. For this small change, neglecting higher order terms in the Taylor series, $i$ th complex eigenvector can be expressed as

$\mathbf{u}_{i}^{(\mathrm{c})} \approx \mathbf{u}_{i}+\left[\nabla \mathbf{u}_{i}\right] \Delta \mathbf{g}$

where $\mathbf{u}_{i}^{(\mathrm{c})}$ denotes the changed complex eigenvector and the $N \times m$ matrix $\left[\nabla \mathbf{u}_{i}\right]=\left[\mathbf{u}_{i, 1}, \mathbf{u}_{i, 2}, \ldots, \mathbf{u}_{i, m}\right]$ with 
$\mathbf{u}_{i, j}=\partial \mathbf{u}_{i} / \partial g_{j}$. In general, $\mathbf{u}_{i, j}$ is a complex vector and can be expanded in terms of the undamped eigenvectors $\mathbf{x}_{k}$, $\forall k=1, \ldots, N$, which form a complete set of basis vectors. Thus, we write

$\mathbf{u}_{i, j}=\sum_{k=1}^{N} a_{i j k} \mathbf{x}_{k}$,

where $a_{i j k}, \forall k=1, \ldots, N$, are set of complex constants to be determined.

For notational convenience, we rewrite Eq. (3) as

$\mathbf{F}_{i} \mathbf{u}_{i}=0$,

where the regular matrix pencil

$\mathbf{F}_{i} \equiv \mathbf{F}\left(\lambda_{i}, \mathbf{g}\right)=-\lambda_{i}^{2} \mathbf{M}+\mathrm{i} \lambda_{i} \mathbf{C}+\mathbf{K}$.

Differentiating Eq. (16), one obtains

$\mathbf{F}_{i} \mathbf{u}_{i, j}=-\mathbf{F}_{i, j} \mathbf{u}_{i}$,

which in view of Eq. (15) can be written as

$\sum_{k=1}^{N} \mathbf{F}_{i} a_{i j k} \mathbf{x}_{k}=-\mathbf{F}_{i, j} \mathbf{u}_{i}$.

Now, without loss of generality, we can define a set of complex variables $b_{i j k}(z), \forall k=1, \ldots, N$, such that

$\sum_{l=1}^{N} \mathbf{F}(z) b_{i j l}(z) \mathbf{x}_{l}=-\mathbf{F}_{i, j} \mathbf{u}_{i}$

when $z=\lambda_{i}$. The term $\mathbf{F}(z)$ appearing in the above equation is the regular matrix pencil defined by $\mathbf{F}(z)=-z^{2} \mathbf{M}+\mathrm{i} z \mathbf{C}+\mathbf{K}$. The relationship between Eqs. (19) and (20) is that

$\left.\mathbf{F}(z)\right|_{\left(z=\lambda_{i}\right)} \equiv \mathbf{F}_{i}, \quad$ and $\left.\quad b_{i j l}(z)\right|_{\left(z=\lambda_{i}\right)} \equiv a_{i j l}$.

Thus, Eq. (20) allows a more general treatment of Eq. (19) because it is valid at all complex frequency points $z$, and Eq. (19) appears as the special case when $z=\lambda_{i}$. Define also the sum

$\mathbf{S}_{u}(z)=\sum_{k=1}^{N} b_{i j k}(z) \mathbf{x}_{k}$

so that from Eq. (15), $\left.\mathbf{u}_{i, j} \equiv \mathbf{S}_{u}(z)\right|_{\left(z=\lambda_{i}\right)}$. A confusion may arise from the term on the right-hand side appearing in the Eqs. (19) and (20): strictly speaking, it should also be described in terms of the general (complex) frequency variable $z$, so that instead of $\mathbf{F}_{i, j}$, it should be written as $\mathbf{F}(z)_{j}$. However, since in the end we will substitute back $z=\lambda_{i}$ to obtain $a_{i j k}$, the use of $\mathbf{F}_{i, j}$ is just a matter of notation and we keep track of it by writing $b_{i j l}(z)$ on the right-hand side of Eq. (20).

Now, premultiplying Eq. (20) by $\mathbf{x}_{k}^{\mathrm{T}}$ and using the orthogonal properties of the undamped mode shapes described by Eq. (5), one obtains the scalar equation

$$
\left(\omega_{k}^{2}-z^{2}+\mathrm{i} z C_{k k}^{\prime}\right) b_{i j k}+\mathrm{i} z \sum_{l \neq k}^{N} C_{k l}^{\prime} b_{i j l}=-\left(\mathbf{x}_{k}^{\mathrm{T}} \mathbf{F}_{i, j} \mathbf{u}_{i}\right) .
$$

For all $k=1,2, \ldots, N$, the above equation can be cast in to a matrix form

$[\mathbf{P}+\mathrm{i} z \mathbf{Q}] \mathbf{b}=\mathbf{R}$,

where $\mathbf{P}=\operatorname{diag}\left[\omega_{k}^{2}-z^{2}+\mathrm{i} z C_{k k}^{\prime}\right]$ and $\mathbf{Q}$ is the matrix $\mathbf{C}^{\prime}$ with all diagonal terms omitted. The vector $\mathbf{R}=$ $-\left\{\mathbf{x}_{1}^{\mathrm{T}} \mathbf{F}_{i, j} \mathbf{u}_{i}, \mathbf{x}_{2}^{\mathrm{T}} \mathbf{F}_{i, j} \mathbf{u}_{i}, \ldots, \mathbf{x}_{i}^{\mathrm{T}} \mathbf{F}_{i, j} \mathbf{u}_{i}\right\}^{\mathrm{T}}$ is known and the vector $\mathbf{b}=\left\{b_{i j 1}(z), b_{i j 2}(z), \ldots, b_{i j N}(z)\right\}^{\mathrm{T}}$ is to be determined. One can, in principle, carry out the associated matrix inversion and subsequently calculate $a_{i j k}$ to obtain $\mathbf{u}_{i, j}$. However, this would be a somewhat expensive numerical exercise and may not offer much physical insight to the analyst. Instead, we seek for an approximate solution by making use of a small damping assumption. Recently, Woodhouse [16] has used this kind of approximate analysis in order to determine transfer functions of systems with general linear damping models. After neglecting the higher order terms in $\mathbf{Q}$, from Eq. (24), one obtains

$\mathbf{b} \approx\left[\mathbf{P}^{-1}-\mathrm{i} z \mathbf{P}^{-1} \mathbf{Q} \mathbf{P}^{-1}\right] \mathbf{R}$.

The $k$ th row of this equation leads to

$$
\begin{aligned}
b_{i j k}(z) \approx & \frac{-\mathbf{x}_{k}^{\mathrm{T}} \mathbf{F}_{i, j} \mathbf{u}_{i}}{\omega_{k}^{2}-z^{2}+\mathrm{i} z C_{k k}^{\prime}} \\
& -\mathrm{i} z \sum_{l \neq k}^{N} \frac{C_{k l}^{\prime}\left(-\mathbf{x}_{l}^{\mathrm{T}} \mathbf{F}_{i, j} \mathbf{u}_{i}\right)}{\left(\omega_{k}^{2}-z^{2}+\mathrm{i} z C_{k k}^{\prime}\right)\left(\omega_{l}^{2}-z^{2}+\mathrm{i} z C_{l l}^{\prime}\right)} .
\end{aligned}
$$

The substitution of $b_{i j k}(z)$ in Eq. (22) and the use of the factorization scheme described by Eq. (11) yields

$$
\begin{aligned}
\mathbf{S}_{u}(z) \approx & \sum_{k=1}^{N} \frac{\left(-\mathbf{x}_{k}^{\mathrm{T}} \mathbf{F}_{i, j} \mathbf{u}_{i}\right) \mathbf{x}_{k}}{-\left(z-\lambda_{k}\right)\left(z+\lambda_{k}^{*}\right)} \\
& -\mathrm{i} z \sum_{k=1}^{N} \sum_{l \neq k}^{N} \frac{C_{k l}^{\prime}\left(-\mathbf{x}_{l}^{\mathrm{T}} \mathbf{F}_{i, j} \mathbf{u}_{i}\right) \mathbf{x}_{k}}{\left(z-\lambda_{k}\right)\left(z+\lambda_{k}^{*}\right)\left(z-\lambda_{l}\right)\left(z+\lambda_{l}^{*}\right)} .
\end{aligned}
$$

This expression has simple poles at $z=\lambda_{k}$ and $z=$ $-\lambda_{k}^{*}, \forall k=1,2, \ldots, N$. The residue of the pole at $z=$ $\lambda_{k}$ can be written as

$$
\begin{aligned}
\operatorname{Res}\left[z=\lambda_{k}\right]= & \frac{\left(-\mathbf{x}_{k}^{\mathrm{T}} \mathbf{F}_{i, j} \mathbf{u}_{i}\right) \mathbf{x}_{k}}{-\left(\lambda_{k}+\lambda_{k}^{*}\right)} \\
& -\mathrm{i} \lambda_{k} \sum_{l \neq k}^{N} \frac{C_{k l}^{\prime}\left(-\mathbf{x}_{l}^{\mathrm{T}} \mathbf{F}_{i, j} \mathbf{u}_{i}\right) \mathbf{x}_{k}+C_{l k}^{\prime}\left(-\mathbf{x}_{k}^{\mathrm{T}} \mathbf{F}_{i, j} \mathbf{u}_{i}\right) \mathbf{x}_{l}}{\left(\lambda_{k}+\lambda_{k}^{*}\right)\left(\lambda_{k}-\lambda_{l}\right)\left(\lambda_{k}+\lambda_{l}^{*}\right)} .
\end{aligned}
$$

Having in mind that $\lambda_{k}+\lambda_{k}^{*} \approx 2 \omega_{k}$ and taking advantage of the symmetry of $C_{k l}^{\prime}$, this equation gives 


$$
\begin{aligned}
& \operatorname{Res}\left[z=\lambda_{k}\right] \\
& \approx \frac{1}{2 \omega_{k}}\left[\left(\mathbf{x}_{k}^{\mathrm{T}} \mathbf{F}_{i, j} \mathbf{u}_{i}\right) \mathbf{x}_{k}\right. \\
& \left.+\sum_{l \neq k}^{N} \frac{\mathrm{i} \lambda_{k} C_{k l}^{\prime}\left\{\left(-\mathbf{x}_{l}^{\mathrm{T}} \mathbf{F}_{i, j} \mathbf{u}_{i}\right) \mathbf{x}_{k}+\left(\mathbf{x}_{k}^{\mathrm{T}} \mathbf{F}_{i, j} \mathbf{u}_{i}\right) \mathbf{x}_{l}\right\}}{\left(\lambda_{k}-\lambda_{l}\right)\left(\lambda_{k}+\lambda_{l}^{*}\right)}\right]
\end{aligned}
$$

Since we neglect the second-order terms involving $C_{k l}^{\prime}, \forall k \neq l$, the above equation can be cast in the form

$$
\begin{aligned}
\operatorname{Res}\left[z=\lambda_{k}\right] \approx \frac{1}{2 \omega_{k}} & {\left[\left\{\mathbf{x}_{k}^{\mathrm{T}}+\sum_{l \neq k}^{N} \frac{\mathrm{i} \lambda_{k} C_{k l}^{\prime} \mathbf{x}_{l}^{\mathrm{T}}}{\left(\lambda_{k}-\lambda_{l}\right)\left(\lambda_{k}+\lambda_{l}^{*}\right)}\right\}_{1 \times N}\right.} \\
& \left.\times\left\{\mathbf{F}_{i, j} \mathbf{u}_{i}\right\}_{N \times 1}\left\{\mathbf{x}_{k}+\sum_{l \neq k}^{N} \frac{\mathrm{i} \lambda_{k} C_{k l}^{\prime} \mathbf{x}_{l}}{\left(\lambda_{k}-\lambda_{l}\right)\left(\lambda_{k}+\lambda_{l}^{*}\right)}\right\}\right] .
\end{aligned}
$$

Now, the use of Eq. (13) for the approximate complex mode shapes reduces this equation to

$\operatorname{Res}\left[z=\lambda_{k}\right] \approx \frac{1}{2 \omega_{k}}\left(\mathbf{u}_{k}^{\mathrm{T}} \mathbf{F}_{i, j} \mathbf{u}_{i}\right) \mathbf{u}_{k}$.

Similarly, the residue of the pole at $z=-\lambda_{k}^{*}$ can be obtained as

$\operatorname{Res}\left[z=-\lambda_{k}^{*}\right] \approx-\frac{1}{2 \omega_{k}}\left(\mathbf{u}_{k}^{* \mathrm{~T}} \mathbf{F}_{i, j} \mathbf{u}_{i}\right) \mathbf{u}_{k}^{*}$

From the residue at these two poles, the series sum in Eq. (27) can be written

$$
\begin{aligned}
\mathbf{S}_{u}(z) & \approx \sum_{k=1}^{N} \frac{\operatorname{Res}\left[z=\lambda_{k}\right]}{z-\lambda_{k}}+\frac{\operatorname{Res}\left[z=-\lambda_{k}^{*}\right]}{z+\lambda_{k}^{*}} \\
& \approx \sum_{k=1}^{N} \frac{1}{2 \omega_{k}}\left[\frac{\left(\mathbf{u}_{k}^{\mathrm{T}} \mathbf{F}_{i, j} \mathbf{u}_{i}\right) \mathbf{u}_{k}}{z-\lambda_{k}}-\frac{\left(\mathbf{u}_{k}^{* \mathrm{~T}} \mathbf{F}_{i, j} \mathbf{u}_{i}\right) \mathbf{u}_{k}^{*}}{z+\lambda_{k}^{*}}\right] .
\end{aligned}
$$

Again from Eq. (22), equating term by term, it can be said that

$b_{i j k}(z) \mathbf{x}_{k} \approx \frac{1}{2 \omega_{k}}\left[\frac{\left(\mathbf{u}_{k}^{\mathrm{T}} \mathbf{F}_{i, j} \mathbf{u}_{i}\right) \mathbf{u}_{k}}{z-\lambda_{k}}-\frac{\left(\mathbf{u}_{k}^{* \mathrm{~T}} \mathbf{F}_{i, j} \mathbf{u}_{i}\right) \mathbf{u}_{k}^{*}}{z+\lambda_{k}^{*}}\right]$.

Substitution of $z=\lambda_{i}$ and use of Eq. (21) leads to

$a_{i j k} \mathbf{x}_{k} \approx \frac{1}{2 \omega_{k}}\left[\frac{\left(\mathbf{u}_{k}^{\mathrm{T}} \mathbf{F}_{i, j} \mathbf{u}_{i}\right) \mathbf{u}_{k}}{\lambda_{i}-\lambda_{k}}-\frac{\left(\mathbf{u}_{k}^{* \mathrm{~T}} \mathbf{F}_{i, j} \mathbf{u}_{i}\right) \mathbf{u}_{k}^{*}}{\lambda_{i}+\lambda_{k}^{*}}\right]$.

It is clear that this expression involving $a_{i j k}$ is not valid for $k=i$. This special case will be considered separately later in this section. After some algebraic operation, the terms appearing within the brackets can further be simplified

$$
\begin{aligned}
\mathbf{u}_{k}^{\mathrm{T}} \mathbf{F}_{i, j} \mathbf{u}_{i} & =\left(1-\gamma_{k i}\right) \mathbf{u}_{k}^{\mathrm{T}} \tilde{\mathbf{F}}_{i, j} \mathbf{u}_{i} \text { and } \\
\mathbf{u}_{k}^{*} \mathbf{F}_{i, j} \mathbf{u}_{i} & =\left(1-\bar{\gamma}_{k i}\right) \mathbf{u}_{k}^{* \mathrm{~T}} \tilde{\mathbf{F}}_{i, j} \mathbf{u}_{i} .
\end{aligned}
$$

Here, the ratios

$$
\begin{aligned}
\gamma_{k i} & =\frac{\mathbf{u}_{k}^{\mathrm{T}}\left(\lambda_{i}^{2} \mathbf{M}+\mathbf{K}\right) \mathbf{u}_{i}}{\mathbf{u}_{i}^{\mathrm{T}}\left(\lambda_{i}^{2} \mathbf{M}+\mathbf{K}\right) \mathbf{u}_{i}} \text { and } \\
\bar{\gamma}_{k i} & =\frac{\mathbf{u}_{k}^{*^{\mathrm{T}}}\left(\lambda_{i}^{2} \mathbf{M}+\mathbf{K}\right) \mathbf{u}_{i}}{\mathbf{u}_{i}^{\mathrm{T}}\left(\lambda_{i}^{2} \mathbf{M}+\mathbf{K}\right) \mathbf{u}_{i}}
\end{aligned}
$$

with

$\tilde{\mathbf{F}}_{i, j}=\left[\mathbf{K}_{, j}-\lambda_{i}^{2} \mathbf{M}_{, j}+\mathrm{i} \lambda_{i} \mathbf{C}_{, j}\right]$

is the matrix which arises only due to the change of the system property matrices with respect to the design parameters. This simplification turns out to be useful, as will be seen in Section 4. Now, by taking the transpose of Eq. (35) and then post-multiplying it by $\mathbf{M} \mathbf{x}_{k}$, and also from the expansion of the complex modes in Eq. (13) noting the fact that $\mathbf{u}_{k}^{\mathrm{T}} \mathbf{M} \mathbf{x}_{k}=\mathbf{u}_{k}^{*^{\mathrm{T}}} \mathbf{M} \mathbf{x}_{k}=1$, we finally obtain

$a_{i j k} \approx \frac{1}{2 \omega_{k}}\left[\frac{\left(1-\gamma_{k i}\right) \mathbf{u}_{k}^{\mathrm{T}} \tilde{\mathbf{F}}_{i, j} \mathbf{u}_{i}}{\lambda_{i}-\lambda_{k}}-\frac{\left(1-\bar{\gamma}_{k i}\right) \mathbf{u}_{k}^{* \mathrm{~T}} \tilde{\mathbf{F}}_{i, j} \mathbf{u}_{i}}{\lambda_{i}+\lambda_{k}^{*}}\right]$,

for $k \neq i$.

In passing, we note that for the undamped case $\gamma_{k i}, \bar{\gamma}_{k i} \rightarrow 0$, and this equation reduces exactly to its corresponding equation (see Eq. (20) in Ref. [6]) for the undamped case.

To obtain $a_{i j i}$, we begin with the expansion of the complex modes in Eq. (6) and obtain the relationship

$\mathbf{u}_{i}^{\mathrm{T}} \mathbf{M} \mathbf{u}_{i}=1+\sum_{l \neq i} \alpha_{l}^{(i)} \alpha_{l}^{(i)} \approx 1$

Differentiation of the above equation with respect to $g_{j}$ together with the symmetry property of $\mathbf{M}$ results in a scalar equation

$\mathbf{u}_{i}^{\mathrm{T}} \mathbf{M} \mathbf{u}_{i, j} \approx-\frac{1}{2}\left(\mathbf{u}_{i}^{\mathrm{T}} \mathbf{M}_{, j} \mathbf{u}_{i}\right)$.

Now, substitution of $\mathbf{u}_{i, j}$ from Eq. (15) yields

$a_{i j i} \approx-\frac{1}{2}\left(\mathbf{u}_{i}^{\mathrm{T}} \mathbf{M}_{, j} \mathbf{u}_{i}\right)-\sum_{k \neq i} \alpha_{k}^{(i)} a_{i j k}$.

Finally, from the above equation together with Eqs. (15) and (39), we obtain

$$
\begin{gathered}
\mathbf{u}_{i, j} \approx-\frac{1}{2}\left(\mathbf{u}_{i}^{\mathrm{T}} \mathbf{M}_{j} \mathbf{u}_{i}\right) \mathbf{x}_{i}+\sum_{k \neq i}^{N} \frac{1}{2 \omega_{k}}\left[\frac{\left(1-\gamma_{k i}\right) \mathbf{u}_{k}^{\mathrm{T}} \tilde{\mathbf{F}}_{i, j} \mathbf{u}_{i}}{\lambda_{i}-\lambda_{k}}\right. \\
\left.-\frac{\left(1-\bar{\gamma}_{k i}\right) \mathbf{u}_{k}^{\mathrm{*}} \tilde{\mathbf{F}}_{i, j} \mathbf{u}_{i}}{\lambda_{i}+\lambda_{k}^{*}}\right]\left(\mathbf{x}_{k}-\alpha_{k}^{(i)} \mathbf{x}_{i}\right) .
\end{gathered}
$$

This is the generalization of the known expression of rates of change of eigenvectors for an undamped system to that of a damped system. Since in Eqs. (8) and (13) the complex eigensolutions are approximately expressed in terms of the undamped eigensolutions, $\mathbf{u}_{i, j}$ derived 
above in turn can be calculated form the undamped eigensolutions only. This offers significant computational benefit as well as ample physical insight since we have avoided the state-space method and utilized the familiar undamped modes.

\section{Total change of eigenvalues and eigenvectors}

We envisage a situation when all the elements of stiffness, mass and damping matrices undergo small changes. This kind of situation may arise when one applies numerical methods to study the sensitivity of the problem. For example, by using the finite element method, any change in some design variable will result in some changes in the stiffness, mass and damping matrices. It may be noted that this kind of numerical study becomes unavoidable when we consider a general problem in which an explicit relationship between some design variable of interest and the system property matrices cannot be obtained easily. Moreover, due to recent developments in stochastic finite element methods and reliability based analysis, the need for random perturbation of the system property matrices also arises. In this section, we generalize the results obtained in the earlier sections to handle wide range of such different problems.

Unlike the previous section, instead of incorporating some specific variables, here all the elements of stiffness, mass and damping matrices become independent variables; thus, we have

$$
\begin{gathered}
\mathbf{u}_{i} \equiv \mathbf{u}_{i}\left(K_{11}, K_{12}, \ldots, K_{N N}, M_{11}, M_{12}, \ldots, M_{N N},\right. \\
\left.C_{11}, C_{12}, \ldots, C_{N N}\right) .
\end{gathered}
$$

From the above equation, after neglecting the higher order terms in the Taylor series the total change in the $j$ th element of the $i$ th eigenvector can be written as

$$
\begin{aligned}
\mathrm{d} U_{j i} \approx & \sum_{r=1}^{N} \sum_{s=1}^{N} \frac{\partial U_{j i}}{\partial K_{r s}} \mathrm{~d} K_{r s}+\sum_{r=1}^{N} \sum_{s=1}^{N} \frac{\partial U_{j i}}{\partial M_{r s}} \mathrm{~d} M_{r s} \\
& +\sum_{r=1}^{N} \sum_{s=1}^{N} \frac{\partial U_{j i}}{\partial C_{r s}} \mathrm{~d} C_{r s} .
\end{aligned}
$$

Substituting $\tilde{\mathbf{F}}_{i, j}$ from Eq. (38) into Eq. (43), the partial derivatives $\partial U_{j i} / \partial K_{r s}, \partial U_{j i} / \partial M_{r s}$ and $\partial U_{j i} / \partial C_{r s}$ can be obtained by setting $g_{j}$ to one of the elements at a time. Doing so, and using the standard notation of matrices, that is $U_{j i}$ denoting $j$ th element of the $i$ th complex eigenvector $\mathbf{u}_{i}$, it can be shown that

$$
\begin{aligned}
& \frac{\partial U_{j i}}{\partial K_{r s}} \approx \sum_{k \neq i} \frac{1}{2 \omega_{k}}\left[\frac{\left(1-\gamma_{k i}\right) U_{r k} U_{s i}}{\lambda_{i}-\lambda_{k}}-\frac{\left(1-\bar{\gamma}_{k i}\right) U_{r k}^{*} U_{s i}}{\lambda_{i}+\lambda_{k}^{*}}\right] \\
& \times\left(X_{j k}-\alpha_{k}^{(i)} X_{j i}\right), \\
& \frac{\partial U_{j i}}{\partial M_{r s}} \approx-\frac{1}{2}\left(U_{r i} U_{s i}\right) U_{j i}-\lambda_{i}^{2} \frac{\partial U_{j i}}{\partial K_{r s}}, \\
& \frac{\partial U_{j i}}{\partial C_{r s}} \approx \mathrm{i} \lambda_{i} \frac{\partial U_{j i}}{\partial K_{r s}} .
\end{aligned}
$$

The above set of equations are easily amenable for computer programming. For the undamped case, similar expressions were introduced by Collins and Thomson [18] based on the results of Fox and Kapoor [1] and are now in extensive use. Another useful fact emerges from this order of approximate analysis: from Eqs. (8) and (13), complex eigenvalues and eigenvectors, and consequently, from Eq. (45), the change of eigenvectors, can be obtained from the undamped eigenvalues and eigenvectors. The undamped eigensolutions are now readily available from commercial software packages (for example FE-packages), and thus using existing resources, it is possible to study the change of eigenvectors for nonproportionally damped structures in an integrated way by varying mass, stiffness and damping matrices all together at a time.

\section{Examples}

\subsection{Rates of change of eigenvalues in a curve veering problem}

A simple two degree-of-freedom system has been considered to illustrate a possible use of the expressions developed so far. Fig. 1 shows the example taken together with the numerical values. When eigenvalues are plotted versus a system parameter they create family of 'root loci'. When two loci approach together they may cross or rapidly diverge. The later case is called 'curve veering'. It is known that during veering, rapid changes take place in the eigensolutions and can be an interesting problem for applying the general results derived in this paper. The system matrices for the example taken can be expressed by

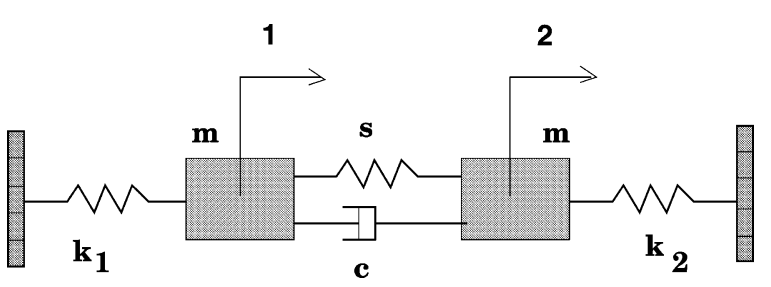

Fig. 1. Two degrees of system show veering, $m=1 \mathrm{~kg}, k_{1}=$ $1000 \mathrm{~N} / \mathrm{m}, s=100 \mathrm{~N} / \mathrm{m}, c=3.0 \mathrm{~N} \mathrm{~s} / \mathrm{m}$. 


$$
\begin{aligned}
& \mathbf{M}=\left[\begin{array}{ll}
m & 0 \\
0 & m
\end{array}\right], \quad \mathbf{C}=\left[\begin{array}{cc}
c & -c \\
-c & c
\end{array}\right], \\
& \mathbf{K}=\left[\begin{array}{cc}
k_{1}+s & -s \\
-s & k_{2}+s
\end{array}\right] .
\end{aligned}
$$

We have focused our attention to calculate the rates of change of eigenvectors with respect to the parameter $s$ which can be obtained from Eq. (43). Complex modes and frequencies appearing in this equation are obtained from the procedure outlined in Section 2. The term $\tilde{\mathbf{F}}_{i, j}$ in this context turns out to be $\mathrm{d} \tilde{\mathbf{F}}_{i} / \mathrm{d} s$ and can be obtained as

$\frac{\mathrm{d} \tilde{\mathbf{F}}_{i}}{\mathrm{~d} s}=\frac{\mathrm{d} \mathbf{K}_{i}}{\mathrm{~d} s}-\lambda_{i}^{2} \frac{\mathrm{d} \mathbf{M}}{\mathrm{d} s}+\mathrm{i} \lambda_{i} \frac{\mathrm{d} \mathbf{C}}{\mathrm{d} s}=\left[\begin{array}{cc}1 & -1 \\ -1 & 1\end{array}\right]$

since $\mathrm{d} \mathbf{M} / \mathrm{d} s=\mathrm{d} \mathbf{C} / \mathrm{d} s=0$. The real part of the derivative of first eigenvector with respect to $s$ for both the coordinates is shown in Fig. 2. The real part has been chosen to be plotted here because a change in stiffness is expected to contribute a significant change in the real part. Derivative of the corresponding real eigenvector for the undamped system (i.e., removing the damper) calculated from the expression derived by Fox and Kapoor [1] is also shown in the same figure (see the figure legend for details). Similar plots for the second eigenvector are shown in Fig. 3. The higher value of the derivatives around the veering range $\left(k_{2} / k_{1} \approx 1\right)$ indicates that eigenvectors become more sensitive to the parameter changes in this region. It is interesting to note that the derivative of the undamped modes and that of the real part of the complex modes almost trace each other except around the veering range, i.e., $0.5<k_{2} / k_{1}<1.5$, where the derivative of the undamped modes show higher values. To get more insight into the results, it is helpful to look at the variation of the modal $Q$-factors

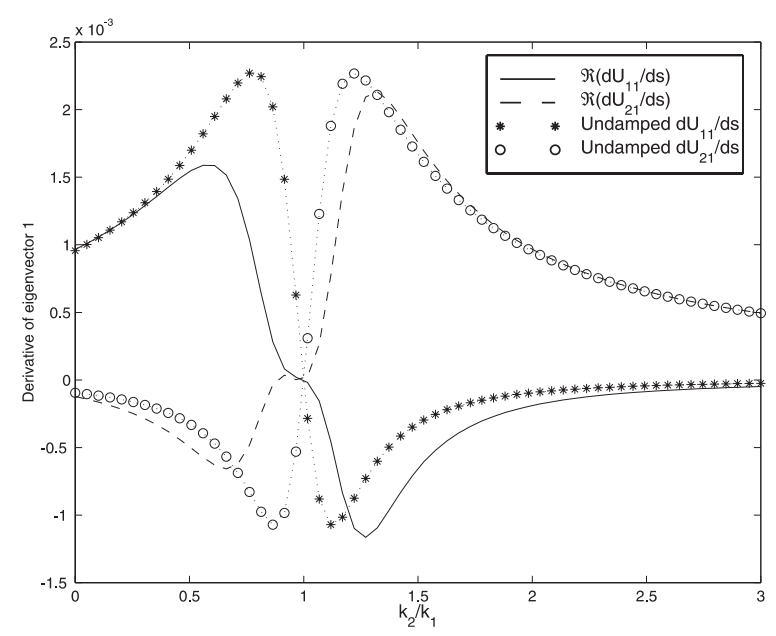

Fig. 2. Real part of the derivative of both the coordinates of the first mode with respect to the stiffness parameter $s$.

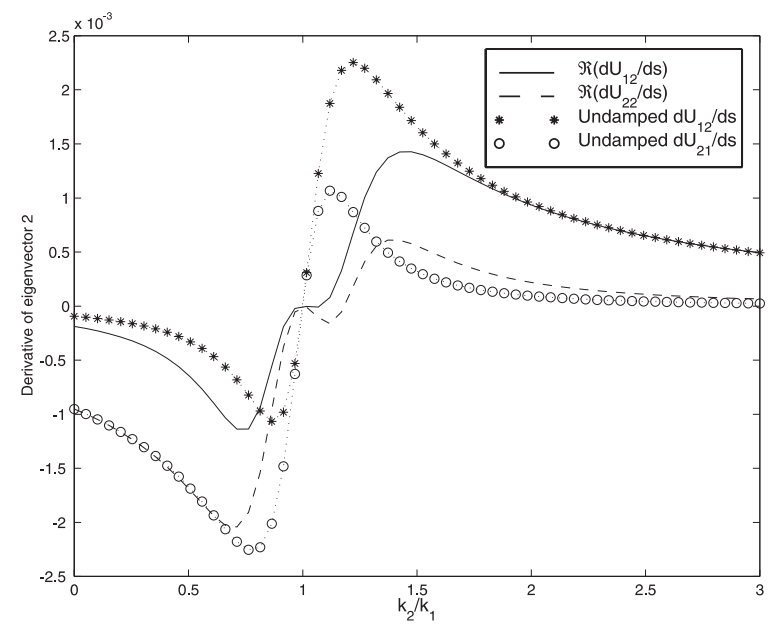

Fig. 3. Real part of the derivative of both the coordinates of the second mode with respect to the stiffness parameter $s$.

for the two modes shown in Fig. 4. For the first mode, $Q$-factor is quite high (in the order of $\approx 10^{3}$ ) near the veering range but still the derivative of the undamped mode and that of the real part of the complex mode for both the coordinates are quite different. Again, away from the veering range, $k_{2} / k_{1}>2$, the $Q$-factor is low but the derivative of the undamped mode and that of real part of the complex mode are quite similar. This is opposite to what we normally expect, as the common belief is that, when the $Q$-factors are high, that is modal dampings are less, the undamped modes and real part of complex modes should behave similarly and vice versa. For the second mode, the $Q$-factor does not change very much due to a parameter variation of $k_{2}$ except that it becomes bit lower around the veering range. But the difference between the sensitivities of the undamped

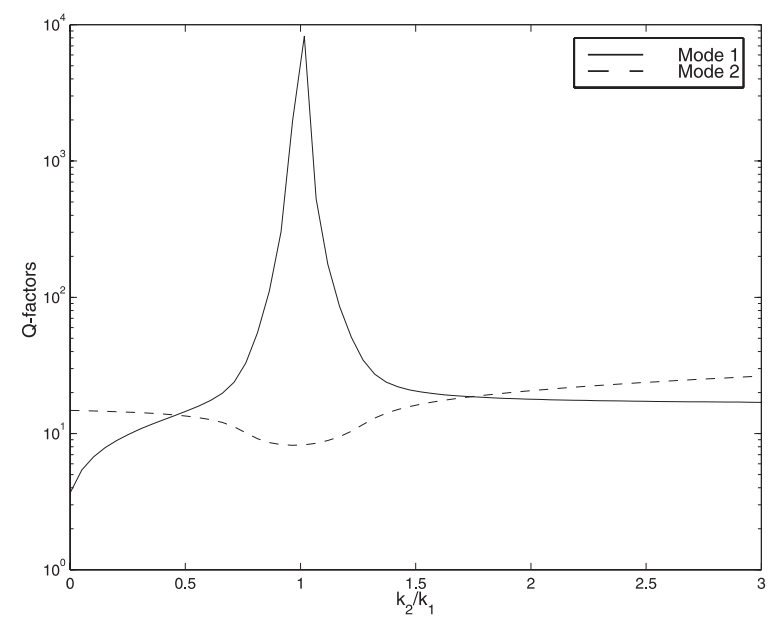

Fig. 4. $Q$-factors for both the modes. 
mode and that of real part of the complex mode for both the coordinates changes much more significantly than the $Q$-factor. This shows that even when the $Q$-factors are similar, the sensitivity of the undamped modes and that the of real part of the complex modes can be significantly different. Thus use of the expression for derivatives of undamped mode shapes can lead to a significant error even when the damping is very low, and for any kind of study involving such a sensitivity analysis the expressions derived in this paper should be used.

\subsection{Numerical accuracy of rates of change of eigenvectors}

The rates of change of eigenvectors are derived by using the small damping assumption and the accuracy associated with it must be judged. However, presently there is no 'exact' method available to determine the rates of change of complex eigenvectors in 'N'-space by which one can verify the accuracy of the expression given by Eq. (43). So, we compare the total change of the eigenvectors when the elements of the system property matrices are given a small perturbation. This in turn can be obtained from Eqs. (45) and (46). It may be noted that the error obtained from this analysis will be more than that given by Eq. (43), since the effect of neglecting the higher order terms of the Taylor series will also be added up. In this way, we can obtain an idea for the upper bound of the error associated with Eq. (43).

We consider a sample problem of a linear array of 10 spring-mass elements (see Fig. 5) with dampers attached from the third to the seventh masses (with the same damping value for all, say $c$ ) and connected to the ground. All the 10 masses and 11 stiffness values are assumed to be unity while we vary the value of the damping $c$. As the dampers are attached only from the third to the seventh masses, the modal matrix becomes non-diagonal resulting in complex modes. We consider a positive change of $2 \%$ for all the values of mass, stiffness and damping, and the exact solutions are obtained by calculating the complex eigenvectors using the statespace method for the changed values of the parameters. For the $2 \%$ change of the system property matrices, the same are again calculated by obtaining the changes from Eqs. (45) and (46) and then adding it with the unperturbed values. Doing so, we also have used the expression for the approximate complex modes derived earlier in Section 2 and, thus, have made use of all the approximations incorporated in this paper. Finally, sub-

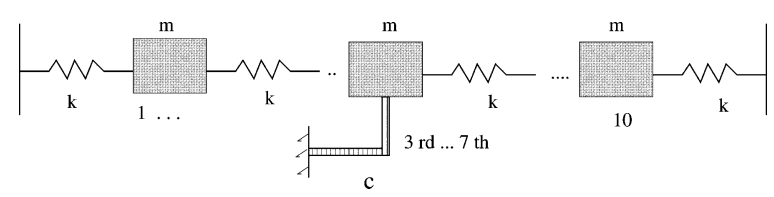

Fig. 5. Linear array of 10 spring-mass oscillators, $m=1, k=1$.

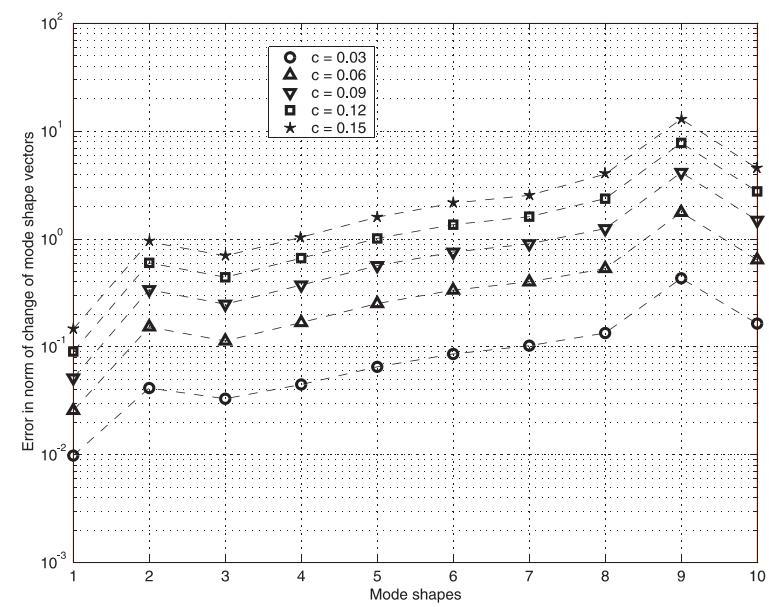

Fig. 6. Norm of $\%$ error in total change of mode shape vectors for a linear array of 10 spring-mass system.

tracting the vectors obtained by these two methods, the error vector can be obtained. In Fig. 6, the norm of this error vector as a percentage of the norm of undamped modes shapes (i.e., for the $i$ th mode, $\left(\| \mathbf{u}_{i}^{\text {exact}}\right.$ $\left.\mathbf{u}_{i}^{\text {approx }}\|/\| \mathbf{x}_{i} \|\right) \times 100$ ) is plotted for all the 10 modes. The value of $c$ is being varied here from 0.03 to 0.15 in steps of 0.03 to see the behaviour of the error as the damping increases. Corresponding associated $Q$-factors for all the modes for the unperturbed system at each values of $c$ are shown in Table 1. This table together with Fig. 6 gives an idea of the error results from the approximate analysis of rate of change of the mode shapes. It may be observed that results are fairly accurate (maximum error $2 \%$ ) for value of $c$ up to 0.09 (see the table for the corresponding $Q$-factors). For $c=0.15$ (the maximum considered here), when the $Q$-factors are quite low, we get a maximum error of about $13 \%$ which is probably not acceptable. By considering such high damping values, we want to see the limit of acceptability of the approximate analysis developed here, in practice, if ever, we encounter such a high value of damping in engineering applications. This analysis indicates that the approximate theory developed here can be used over a wide range of engineering applications, and, as a rule of thumb, results in acceptable accuracy when $Q$-factors are above 10 or so. This conclusion is based on the sample problem being studied here: there might be special cases when anomalies may be observed, which however needs further investigation.

\section{Conclusion}

Derivatives of complex eigenvectors of non-proportionally damped discrete linear system with respect to 
Table 1

$Q$-factors for the 10 modes at five different values of $c$

\begin{tabular}{lrrrrr}
\hline Mode no. & $c=0.03$ & $c=0.06$ & $c=0.09$ & $c=0.12$ & $c=0.15$ \\
\hline 1 & 24.9106 & 12.4191 & 8.2391 & 6.1369 & 4.8655 \\
2 & 90.5875 & 45.2995 & 30.2060 & 22.6610 & 18.1352 \\
3 & 144.6858 & 72.3829 & 48.2995 & 36.2708 & 29.0638 \\
4 & 151.1147 & 75.5415 & 50.3437 & 37.7399 & 30.1741 \\
5 & 188.0051 & 94.0001 & 62.6635 & 46.9937 & 37.5901 \\
6 & 216.9776 & 108.4985 & 72.3435 & 54.2701 & 43.4299 \\
7 & 235.0960 & 117.4584 & 78.2057 & 58.5487 & 46.7294 \\
8 & 316.9661 & 158.7913 & 106.1986 & 79.9947 & 64.3362 \\
9 & 309.1315 & 155.5264 & 104.7780 & 79.7679 & 65.0720 \\
\end{tabular}

the system parameters have been derived using small damping assumption. The analytical method developed avoids the use of the state-space formulation and is more consistent with the notion of traditional modal analysis. Based on the classical first-order perturbation theory, an expression for the complex eigenvectors is suggested, and it was shown that a minor correction can result in more accuracy. Derivatives of complex eigenvectors were expressed in terms of complex eigensolutions derived earlier in terms of the real undamped modes. Based on this formulation, the total change in eigenvalues and eigenvectors was obtained for a more general case when all the elements of mass, stiffness and damping matrices are varying. The small damping approximation used to derive rates of change of the complex eigenvectors gives an acceptable accuracy within the range of most engineering applications. The expressions derived here generalize the earlier results for undamped systems to the damped systems, and will allow sensitivity studies of eigensolutions by involving mass, stiffness and damping properties to be undertaken in an unified manner using familiar classical normal modes only.

\section{Acknowledgements}

I am indebted to Dr. Jim Woodhouse for his careful reading of the manuscript and helping me to choose the examples. I am also grateful to Professor Robin Langley for his valuable comments on this problem. Finally, I want to thank Dr. Atul Bhaskar of IIT, Delhi, for providing me with Ref. [17].

\section{References}

[1] Fox RL, Kapoor MP. Rates of change of eigenvalues and eigenvectors. AIAA J 1968;6(12):2426-9.
[2] Plaut RH, Huseyin K. Derivatives of eigenvalues and eigenvectors in non-self-adjoint systems. AIAA J 1973; 11(2):250-1.

[3] Nelson RB. Simplified calculation of eigenvector derivatives. AIAA J 1976;14(9):1201-5.

[4] Adelman HM, Haftka RT. Sensitivity analysis of discrete structural system. AIAA J 1986;24(5):823-32.

[5] Murthy DV, Haftka RT. Derivatives of eigenvalues and eigenvectors of a general complex matrix. Int $\mathbf{J}$ Numer Meth Engng 1988;26:293-311.

[6] Caughey TK. Classical normal modes in damped linear dynamic systems. J Appl Mech ASME 1960;27: 269-71.

[7] Sestieri A, Ibrahim SR. Analysis of errors and approximations in the use of modal coordinates. J Sound Vibration 1994;177(2):145-57.

[8] Rogers LC. Derivatives of eigenvalues and eigenvectors. AIAA J 1970;8(5):943-4.

[9] Brandon JA. Derivation and significance of second-order modal design sensitivities. AIAA J 1984;22(5):723-4.

[10] Zeng QH. Highly accurate modal method for calculating eigenvector derivatives in viscous damping systems. AIAA J 1995;33(4):746-51.

[11] Lancaster P. Lambda-matrices and vibrating system. New York: Pergamon Press, 1966.

[12] Caughey TK, O'Kelly MEJ. Classical normal modes in damped linear dynamic systems. J Appl Mech ASME 1965;32:583-8.

[13] Newland DE. Mechanical vibration analysis and computation. New York: Longman, Harlow and Wiley, 1989.

[14] Rayleigh Lord. Theory of sound, vols. 1, 2, 2nd ed. New York: Dover Publications, 1897 (re-issue 1945).

[15] Cronin DL. Eigenvalue and eigenvector determination for non-classically damped dynamic systems. Comp Struct 1990;36(1):133-8.

[16] Woodhouse J. Linear damping models for structural vibration. J Sound Vibration 1998;215(3):547-69.

[17] Bhaskar A. Personal communication. Cambridge, UK. April 1998.

[18] Collins JD, Thomson WT. The eigenvalue problem for structural systems with statistical properties. AIAA J 1969;74(4):642-8. 